\title{
India reaches milestone of no cases of wild poliovirus for 12 months
}

We misspelt the name of one of the authors of this News story (BMJ 2012;344:e1328, doi:10.1136/bmj.e1328). The first author is Gurmeen Kaur [not Kaura].

Cite this as: BMJ 2012;344:e1519

๑ BMJ Publishing Group Ltd 2012 